\title{
Numerical aspects of computing the Moore-Penrose inverse of full column rank matrices
}

\author{
Alicja Smoktunowicz • Iwona Wróbel
}

Received: 27 September 2010 / Accepted: 12 September 2011 / Published online: 14 October 2011

(C) The Author(s) 2011. This article is published with open access at Springerlink.com

\begin{abstract}
This paper presents a comparison of certain direct algorithms for computing the Moore-Penrose inverse, for matrices of full column rank, from the point of view of numerical stability. It is proved that the algorithm using Householder QR decomposition, implemented in floating point arithmetic, is forward stable but only conditionally mixed forward-backward stable. A similar result holds also for the Classical Gram-Schmidt algorithm with reorthogonalization (CGS2). This algorithm was developed and analyzed by Abdelmalek (BIT, 11(4):354-367, 1971) and its detailed error analysis was given in Giraud et al. (Numer. Math. 101(1):87-100, 2005).

We incorporate the recent result of Byers and Xu (SIAM J. Matrix Anal. Appl. 30:822-843, 2008) to show that the algorithm based on the Golub-Kahan bidiagonalization is mixed forward-backward stable under natural assumptions.
\end{abstract}

Keywords The Moore-Penrose inverse - Numerical stability · Condition number . Singular value decomposition (SVD)

Mathematics Subject Classification (2000) 15A09 - 15A12 - 65F25 • 65F05 • $65 \mathrm{G} 50$

\section{Introduction}

In this paper we will give a rounding error analysis of certain algorithms for computing the Moore-Penrose inverse $A^{\dagger}$, of $A \in \mathbb{R}^{m \times n}$, where $A$ has full column rank, i.e.

Communicated by Lars Eldén.

A. Smoktunowicz $(\bowtie) \cdot$ I. Wróbel

Faculty of Mathematics and Information Science, Warsaw University of Technology,

Pl. Politechniki 1, Warsaw 00-661, Poland

e-mail: smok@mini.pw.edu.pl

I. Wróbel

e-mail: wrubelki@wp.pl 
$\operatorname{rank}(A)=n \leq m$. In this case $A^{\dagger}$ is the left inverse of $A$, i.e. $A^{\dagger} A=I_{n}$, where $I_{n}$ denotes the $n \times n$ identity matrix. In our comparison we will take into account mainly numerical stability and algebraic complexity of all considered algorithms.

The Moore-Penrose inverse is a powerful tool in computing polar decomposition, the areas of electrical networks, control theory, filtering, estimation theory and pattern recognition (see, e.g., [3, 13-15]). However, if one wants to solve the least-squares problem it is usually recommended to apply methods which do not compute $A^{\dagger}$ directly but use numerically stable factorization of $A$ instead. For more details on numerical properties of algorithms for the least-squares problems we refer the reader to [2].

In the last years a number of fast algorithms for computing the Moore-Penrose inverse of structured and block matrices have been designed. There is a variety of new papers dealing with numerical algorithms, whose authors neglect the issue of numerical stability of their algorithms and focus only on complexity (number of arithmetic operations). However, very often considered methods are not accurate up to the limitations of data and conditioning of the problem. Numerical properties of main approaches to the problem of computing the Moore-Penrose inverse are not stated explicitly anywhere in the literature.

There are many methods for computing $A^{\dagger}$, some of them of high numerical quality. They are available in standard libraries, such as LAPACK and Matlab. Nevertheless this area of research is very active and some authors propose methods of computing $A^{\dagger}$ based on the formula $A^{\dagger}=\left(A^{T} A\right)^{-1} A^{T}$ (see, e.g., [6, 16-19]), where the matrix $A^{T} A$ is formed explicitly. This algorithm may be fast, compared to the SVD decomposition, it is well known however, that for ill-conditioned matrices this approach may lead to severe loss of accuracy. The standard example is the famous Läuchli matrix, see, e.g. [2, Example 2.2.1], where this approach fails completely even for $3 \times 2$ matrices. This is due to the fact that in this example the computed matrix $A^{T} A$ is singular!

The most commonly used numerically stable algorithm for computing $A^{\dagger}$ is based on the SVD decomposition. The SVD of $A$ is recommended for general rank deficient problems. However in many applications it is too expensive to compute the SVD and one has to apply some numerically stable simpler decompositions. Among these the most important are algorithms based on the $Q R$ decomposition and bidiagonal reductions. When $A$ has full column rank then $Q R$ decompositions with column pivoting might be a method of choice. The SVD is in general the most reliable method for determining the numerical rank of a matrix. However, in practice, the $Q R$ decomposition with column pivoting often works as well and requires less work. However, sometimes, the numerical rank of a matrix computed by some software can be severely underestimated, for details see [8]. Often the first step is to make use of a rank-revealing decomposition (RRD), computing an RRD cheaply. For details see, for example, [5, 11] and [2, pp. 21-23]. Higham [11] developed and analyzed use of the $Q R$ decomposition with complete pivoting (column pivoting with row sorting or row pivoting) to compute the SVD.

There are plenty of bidiagonal reductions as well and this area of research is very rich. Important algorithm for computing the bidiagonal decomposition of $A$ has been developed by Chan [4]. The idea, also mentioned in Lawson and Hanson [18, 
pp. 119, 122] is to first find the thin $Q R$ decomposition of $A$ and, next, to apply the bidiagonal reduction to a triangular factor of the $Q R$ decomposition. The method of Chan is more efficient than the Golub-Kahan bidiagonalization algorithm. For computational details see [4] and [5].

We consider the following algorithms

\section{Algorithm I (Chol)}

- Compute the upper-triangular part of the matrix $M=A^{T} A$.

- Find the Cholesky factorization $M=R^{T} R$.

- Solve the equation $R^{T} R X=A^{T}$ for $X$ (by solving two equations with triangular matrices: $R^{T} Y=A^{T}$ and $R X=Y$ ).

\section{Algorithm II $(Q R)$}

- Find the thin Householder $Q R$ decomposition of $A$, i.e. $A=Q R$, where $Q \in$ $\mathbb{R}^{m \times n}$ is left orthogonal (i.e. $Q^{T} Q=I_{n}$ ) and $R \in \mathbb{R}^{n \times n}$ is upper-triangular. The assumption $\operatorname{rank}(A)=n$ guaranties that $R$ is nonsingular.

- Then $X=A^{\dagger}=R^{-1} Q^{T}$ and it is computed from the equation $R X=Q^{T}$ by back substitution.

\section{Algorithm III ( $\left.Q R_{\text {pivot }}\right)$}

- Find the thin Householder $Q R$ decomposition of $A$, with column pivoting, i.e. $A P=Q R$, where $Q \in \mathbb{R}^{m \times n}$ is left orthogonal (i.e. $Q^{T} Q=I_{n}$ ), $R \in \mathbb{R}^{n \times n}$ is upper-triangular and $P \in \mathbb{R}^{n \times n}$ is a permutation matrix. The assumption $\operatorname{rank}(A)=n$ guaranties that $R$ is nonsingular.

- Then $X=A^{\dagger}=P R^{-1} Q^{T}$ and it is computed in two steps: the equation $R Y=Q^{T}$ is solved for $Y$ by back substitution and then we compute $X=P Y$.

\section{Algorithm IV ( $\left.Q R_{\mathrm{CGS} 2}\right)$}

This algorithm was developed and analyzed by Abdelmalek in [1] and its detailed error analysis was given by Giraud, Langou, Rozložnik, and Van Den Eshof in [9].

- Apply the Classical Gram-Schmidt algorithm with reorthogonalization (CGS2) to produce the $Q R$ decomposition of $A$, i.e. $A=Q R$, where $Q \in \mathbb{R}^{m \times n}$ is left orthogonal (i.e. $Q^{T} Q=I_{n}$ ) and $R \in \mathbb{R}^{n \times n}$ is upper-triangular. The assumption $\operatorname{rank}(A)=n$ guaranties that $R$ is nonsingular.

- Then $X=A^{\dagger}=R^{-1} Q^{T}$ and it is computed from the equation $R X=Q^{T}$ by back substitution.

\section{Algorithm V (Bidiag 1$)$}

- Use Golub-Kahan algorithm to reduce $A$ to a bidiagonal form, i.e. write $A$ as $A=U B V^{T}$, where $U \in \mathbb{R}^{m \times n}$ is left orthogonal (i.e. $U^{T} U=I_{n}$ ), $V \in \mathbb{R}^{n \times n}$ is orthogonal, and $B \in \mathbb{R}^{n \times n}$ is bidiagonal (i.e. the nonzero elements can be located on the main diagonal or on the superdiagonal only). The assumption $\operatorname{rank}(A)=n$ guaranties that $B$ is nonsingular. 
Table 1 Cost of algorithms for computing $X=A^{\dagger}$ for $A \in \mathbb{R}^{m \times n}$ of full column rank

\begin{tabular}{ll}
\hline Algorithm & Number of flops \\
\hline Algorithm I (Cholesky) & $3 m n^{2}+n^{3} / 3$ \\
Algorithm II (Thin Householder $Q R)$ & $5 m n^{2}-4 / 3 n^{3}$ \\
Algorithm V (Bidiagonalization, Golub-Kahan) & $8 m n^{2}-2 / 3 n^{3}$ \\
Algorithm VII (SVD, Golub-Reinsch) & $14 m n^{2}+16 / 3 n^{3}$ \\
\hline
\end{tabular}

- Then $X=A^{\dagger}=V B^{-1} U^{T}$ and it is computed in two steps: first, solve the equation $B Y=U^{T}$ for $Y$ by back substitution, then compute $X=V Y$.

\section{Algorithm VI (Bidiag 2$)$}

- Find the $Q R$ decomposition of $A(A=Q R)$ and bidiagonalize $R$, as described in Algorithm $\mathrm{V}$. We get $R=U B V^{T}$, where $U \in \mathbb{R}^{n \times n}$ is orthogonal (i.e. $U^{T} U=I_{n}$ ), $V \in \mathbb{R}^{n \times n}$ is orthogonal, and $B \in \mathbb{R}^{n \times n}$ is bidiagonal.

- Then $X=A^{\dagger}=V B^{-1}(Q U)^{T}$ and it is computed in two steps: first, solve the equation $B Y=(Q U)^{T}$ for $Y$ by back substitution, then compute $X=V Y$.

\section{Algorithm VII (SVD)}

- Find the thin singular value decomposition of $A$, i.e. $A=U \Sigma V^{T}$, where $U \in$ $\mathbb{R}^{m \times n}$ is left orthogonal (i.e. $U^{T} U=I_{n}$ ), $V \in \mathbb{R}^{n \times n}$ is orthogonal, and $\Sigma \in \mathbb{R}^{n \times n}$ is diagonal. The assumption $\operatorname{rank}(A)=n$ guaranties that $\Sigma$ is nonsingular.

- Then $X=A^{\dagger}=V \Sigma^{-1} U^{T}$.

Table 1 presents computational cost of Algorithms I, II, V and VII counted as a number of flops. By flop we mean any of the elementary scalar operations: $+,-, *, /$. We assume that explicit formation of $Q \in \mathbb{R}^{m \times n}, U \in \mathbb{R}^{m \times n}$ and $V \in \mathbb{R}^{n \times n}$ is used. The costs of decompositions of matrices are taken partly from Higham (see [13, p. 337]).

Less expensive modifications of mentioned methods do not require explicit form of the factors $Q, U$ and $V$. For example, the variant of Byers and $\mathrm{Xu}$ in [3] has the cost $8 m n^{2}-2 n^{3}$ flops instead of $8 m n^{2}-2 / 3 n^{3}$ for Algorithm V.

Algorithm I (Chol) based on the Cholesky decomposition is the cheapest method, however, it is not suitable for every matrix (see Sects. 3-4).

Algorithm V using the Householder bidiagonalization is mixed forward-backward stable for computing the inverses of nonsingular square matrices, which was proved recently by Byers and $\mathrm{Xu}$ in [3]. In the present paper we prove that Algorithms V-VII are also mixed forward-backward stable in sense of (2.7)-(2.8) for $m \times n$ matrices of full column rank. The argument is similar to this of Byers and $\mathrm{Xu}$.

The error analysis of some algorithms for computing the Moore-Penrose inverse is given in Sect. 3. Section 4 is devoted to numerical experiments. We prove by giving some computational examples that Algorithms II and IV are not mixed forwardbackward stable, but only conditionally mixed forward-backward stable, see Theorem 3.2 and Tables 9 and 12 . 


\section{Perturbation theory for the Moore-Penrose inverse}

This section contains several lemmas that will be used in the error analysis in Sect. 3. The first one may be found in [2, p. 14].

Lemma 2.1 Let $A, \Delta A \in \mathbb{R}^{m \times n}, m \geq n$ and let $\sigma_{1}(A) \geq \sigma_{2}(A) \geq \cdots \geq \sigma_{n}(A)$ be the singular values of $A$, and $\sigma_{1}(A+\Delta A) \geq \sigma_{2}(A+\Delta A) \geq \cdots \geq \sigma_{n}(A+\Delta A)$ be the singular values of $A+\Delta A$.

Then

$$
\left|\sigma_{i}(A+\Delta A)-\sigma_{i}(A)\right| \leq\|\Delta A\|_{2}, \quad i=1, \ldots, n .
$$

Next lemma is a corollary to the previous one (see [2, pp. 26-27]). The formula (2.5) is Wedin's result (see [2, Theorem 1.4.4]).

Lemma 2.2 Assume that $A \in \mathbb{R}^{m \times n}, m \geq n=\operatorname{rank}(A)$. Let $\|\Delta A\|_{2} \leq \varepsilon\|A\|_{2}$ and $\varepsilon \kappa_{2}(A)<1$, where $\kappa_{2}(A)$ denotes the condition number of $A$, i.e.

$$
\kappa_{2}(A)=\|A\|_{2}\left\|A^{\dagger}\right\|_{2}=\frac{\sigma_{1}(A)}{\sigma_{n}(A)} .
$$

Then $\operatorname{rank}(A+E)=\operatorname{rank}(A)=n$ and

$$
\begin{aligned}
\|A+\Delta A\|_{2} & \leq(1+\varepsilon)\|A\|_{2}, \\
\left\|(A+\Delta A)^{\dagger}\right\|_{2} & \leq \frac{\left\|A^{\dagger}\right\|_{2}}{1-\varepsilon \kappa_{2}(A)}, \\
\left\|(A+\Delta A)^{\dagger}-A^{\dagger}\right\|_{2} & \leq \sqrt{2} \varepsilon \kappa_{2}(A)\left\|(A+\Delta A)^{\dagger}\right\|_{2}, \\
\frac{\left\|(A+\Delta A)^{\dagger}-A^{\dagger}\right\|_{2}}{\left\|A^{\dagger}\right\|_{2}} & \leq \frac{\sqrt{2} \varepsilon \kappa_{2}(A)}{1-\varepsilon \kappa_{2}(A)} .
\end{aligned}
$$

We will study numerical stability of some basic direct methods for computing the Moore-Penrose inverse $A^{\dagger}$ for $A \in \mathbb{R}^{m \times n}$, where $A$ has full column rank, i.e. $\operatorname{rank}(A)=n \leq m$. In some applications, e.g. in computing polar decomposition of matrices (see [3]), strong property of algorithms for the Moore-Penrose inverse is required, namely mixed forward-backward stability. It means that the computed matrix $\tilde{X}$ is a slightly perturbed generalized inverse of some slightly perturbed matrix $A+\Delta A$.

The precise definition is the following.

Definition 2.1 An algorithm for computing $A^{\dagger}$, where $A \in \mathbb{R}^{m \times n}$, is mixed forwardbackward stable, if the computed result $\tilde{X} \in \mathbb{R}^{n \times m}$ satisfies

$$
\tilde{X}+\Delta X=(A+\Delta A)^{\dagger},
$$

with

$$
\|\Delta A\|_{2} \leq c \varepsilon_{M}\|A\|_{2}, \quad\|\Delta X\|_{2} \leq c \varepsilon_{M}\|\tilde{X}\|_{2},
$$

where $c$ is a small constant depending upon $m$ and $n$, and $\varepsilon_{M}$ is machine precision. 
The result given in (2.6) measures the sensitivity of the Moore-Penrose inverse to small perturbation in the input data $A$. In practice one should use algorithms which are numerically stable, i.e. for which the relative forward error satisfies $\frac{\left\|\tilde{X}-A^{\dagger}\right\|_{2}}{\left\|A^{\dagger}\right\|_{2}}=$ $\mathcal{O}\left(\varepsilon_{M} \kappa_{2}(A)\right)$.

Definition 2.2 We say that an algorithm for computing $A^{\dagger}$, where $A \in \mathbb{R}^{m \times n}$, is forward stable, if the computed result $\tilde{X} \in \mathbb{R}^{n \times m}$ satisfies

$$
\frac{\left\|\tilde{X}-A^{\dagger}\right\|_{2}}{\left\|A^{\dagger}\right\|_{2}} \leq L \varepsilon_{M} \kappa_{2}(A)
$$

where $\kappa_{2}(A)=\left\|A^{\dagger}\right\|_{2}\|A\|_{2}$ is the condition number of $A, L$ is a small constant depending upon $m, n$ and $\varepsilon_{M}$ is machine precision.

Now we show that mixed forward-backward stability implies forward stability. The opposite implication is not true (for examples see Sect. 4).

Theorem 2.1 Assume that $A \in \mathbb{R}^{m \times n}, m \geq n=\operatorname{rank}(A)$. Let $\tilde{X}$ be computed by mixed forward-backward algorithm satisfying (2.7)-(2.8). Suppose that $c \varepsilon_{M} \kappa_{2}(A)<1$.

Then

$$
\frac{\left\|\tilde{X}-A^{\dagger}\right\|_{2}}{\left\|A^{\dagger}\right\|_{2}} \leq \frac{c \varepsilon_{M}}{1-c \varepsilon_{M} \kappa_{2}(A)}\left(\sqrt{2} \kappa_{2}(A)+\frac{1}{1-c \varepsilon_{M}}\right) .
$$

Proof We start by writing

$$
\tilde{X}-A^{\dagger}=\left((A+\Delta A)^{\dagger}-A^{\dagger}\right)-\Delta X .
$$

Taking norms we get

$$
\left\|\tilde{X}-A^{\dagger}\right\|_{2} \leq\left\|(A+\Delta A)^{\dagger}-A^{\dagger}\right\|_{2}+c \varepsilon_{M}\|\tilde{X}\|_{2} .
$$

By (2.5) we obtain, taking $\varepsilon=c \varepsilon_{M}$, that

$$
\left\|(A+\Delta A)^{\dagger}-A^{\dagger}\right\|_{2} \leq \sqrt{2} c \varepsilon_{M} \kappa_{2}(A)\left\|(A+\Delta A)^{\dagger}\right\|_{2} .
$$

Now, since $\tilde{X}=(A+\Delta A)^{\dagger}-\Delta X$, we have

$$
\|\tilde{X}\|_{2} \leq\left\|(A+\Delta A)^{\dagger}\right\|_{2}+\|\Delta X\|_{2} \leq\left\|(A+\Delta A)^{\dagger}\right\|_{2}+c \varepsilon_{M}\|\tilde{X}\|_{2} .
$$

Therefore,

$$
\|\tilde{X}\|_{2} \leq \frac{1}{1-c \varepsilon_{M}}\left\|(A+\Delta A)^{\dagger}\right\|_{2} .
$$

This and (2.10)-(2.11) gives

$$
\left\|\tilde{X}-A^{\dagger}\right\|_{2} \leq c \varepsilon_{M}\left(\sqrt{2} \kappa_{2}(A)+\frac{1}{1-c \varepsilon_{M}}\right)\left\|(A+\Delta A)^{\dagger}\right\|_{2} .
$$

This together with (2.4) implies (2.9). The proof is complete. 
How forward error is related to the normalized residuum? A simple calculation shows the following result.

Lemma 2.3 Assume that $A \in \mathbb{R}^{m \times n}, m \geq n=\operatorname{rank}(A)$. Then for every nonzero matrix $\tilde{X} \in \mathbb{R}^{n \times m}$

$$
\frac{\left\|\tilde{X} A-I_{n}\right\|_{2}}{\|A\|_{2}\|\tilde{X}\|_{2}} \leq \frac{\left\|\tilde{X}-A^{\dagger}\right\|_{2}}{\|\tilde{X}\|_{2}} .
$$

Proof Since $A$ has full column rank, we have $I_{n}=A^{\dagger} A$, and hence $\tilde{X} A-I_{n}=$ $\left(\tilde{X}-A^{\dagger}\right) A$. Taking norms, we get $\left\|\tilde{X} A-I_{n}\right\|_{2} \leq\left\|\tilde{X}-A^{\dagger}\right\|_{2}\|A\|_{2}$. From this the assertion of the lemma follows immediately.

This lemma establishes the fact that the left residual $\frac{\left\|\tilde{X} A-I_{n}\right\|_{2}}{\|A\|_{2}\|\tilde{X}\|_{2}}$ never exceeds the relative error $\frac{\left\|\tilde{X}-A^{\dagger}\right\|_{2}}{\|\tilde{X}\|_{2}}$. Next lemma presents more precise estimation if mixed forward-backward stable algorithm for computing the Moore-Penrose inverse is used.

Lemma 2.4 Assume that $A \in \mathbb{R}^{m \times n}, m \geq n=\operatorname{rank}(A)$. Let $\tilde{X}+\Delta X=(A+\Delta A)^{\dagger}$, where $\|\Delta A\|_{2} \leq \varepsilon\|A\|_{2}$ and $\|\Delta X\|_{2} \leq \varepsilon\|\tilde{X}\|_{2}$. Assume also that $\varepsilon \kappa_{2}(A)<1$.

Then

$$
\frac{\left\|\tilde{X} A-I_{n}\right\|_{2}}{\|A\|_{2}\|\tilde{X}\|_{2}} \leq 2 \varepsilon+\varepsilon^{2} .
$$

Proof Start by writing $\tilde{X} A-I_{n}=\left(\tilde{X}(A+\Delta A)-I_{n}\right)-\tilde{X} \Delta A$. The assumption $\operatorname{rank}(A+\Delta A)=\operatorname{rank}(A)$ implies that $(A+\Delta A)^{\dagger}(A+\Delta A)=I_{n}$. Then $\tilde{X} A-I_{n}=$ $-(\Delta X(A+\Delta A)+\tilde{X} \Delta A)$. Taking norms, we get the desired inequality.

The above lemma shows that if an algorithm is mixed forward-backward stable, then the left residual $\frac{\left\|\tilde{X} A-I_{n}\right\|_{2}}{\|A\|_{2}\|\tilde{X}\|_{2}}$ is of order of machine precision. This fact is used in an example, see Tables 9 and 12 .

\section{Error analysis}

In this section we present error analysis of Algorithms I-VII.

\subsection{Basic lemmas}

First of all we collect basic facts needed in error analysis of these algorithms, incorporating well known stability results on matrix operations.

We will use the following useful inequalities

$$
\|A\|_{2} \leq\||A|\|_{2} \leq \sqrt{\min \{m, n\}}\|A\|_{2},
$$

where $|A|=\left(\left|a_{i j}\right|\right)$ denotes the absolute value of $A=\left(a_{i j}\right) \in \mathbb{R}^{m \times n}$. 
Inequalities between matrices are understood to hold componentwise, i.e. we write $|A| \leq|B|$ for given $A, B \in \mathbb{R}^{m \times n}$ if $\left|a_{i j}\right| \leq\left|b_{i j}\right|$ for all $i=1, \ldots, m$ and $j=1, \ldots, n$. It is known that $|A| \leq|B|$ implies $\||A|\|_{2} \leq\||B|\|_{2}$, see, e.g. [2, pp. 32-33].

The first result describes the influence of the rounding errors on the solution of triangular systems, see [12, pp. 142, 262-263].

Lemma 3.1 Let $R \in \mathbb{R}^{n \times n}$ be a nonsingular upper triangular matrix and let $F \in$ $\mathbb{R}^{n \times m}, n \leq m$. Then the computed solution $\tilde{X} \in \mathbb{R}^{n \times m}$ to $R X=F$ obtained by back substitution satisfies

$$
R \tilde{X}=F+\Delta F, \quad|\Delta F| \leq d_{1} \varepsilon_{M}|R \| \tilde{X}|, \quad \text { and } \quad\|\Delta F\|_{2} \leq n d_{1} \varepsilon_{M}\|R\|_{2}\|\tilde{X}\|_{2}
$$

with $d_{1} \approx n$.

Proof The proof is straightforward. Let $F=\left[f_{1}, f_{2}, \ldots, f_{m}\right]$ and $\tilde{X}=\left[\tilde{x}_{1}, \tilde{x}_{2}\right.$, $\left.\ldots, \tilde{x}_{m}\right]$, where $f_{j}$ and $\tilde{x}_{j}$ denote $j$ th columns of $F$ and $\tilde{X}$, respectively.

It is well known that the computed solution $\tilde{x}_{j}$ to each system $R x_{j}=f_{j}$ satisfies

$$
\left(R+\Delta R_{j}\right) \tilde{x}_{j}=f_{j}, \quad \text { where }\left|\Delta R_{j}\right| \leq d_{1} \varepsilon_{M}|R|, d_{1} \approx n .
$$

From this it follows that $R \tilde{X}=F+\Delta F$, with $\Delta F=-\left[\Delta R_{1} \tilde{x}_{1}, \Delta R_{2} \tilde{x}_{2}, \ldots\right.$, $\left.\Delta R_{m} \tilde{x}_{m}\right]$, so $|\Delta F| \leq d_{1} \varepsilon_{M}|R \| \tilde{X}|$. This gives $\|\Delta F\|_{2} \leq d_{1} \varepsilon_{M}\||R|\|_{2}\||\tilde{X}|\|_{2}$. By (3.1) we get $\||R|\|_{2} \leq \sqrt{n}\|R\|_{2}$ and $\||\tilde{X}|\|_{2} \leq \sqrt{n}\|\tilde{X}\|_{2}$, which implies the desired conclusion.

Lemma 3.2 Let $B \in \mathbb{R}^{n \times n}$ be a nonsingular bidiagonal matrix and let $F \in \mathbb{R}^{n \times m}$. Then the computed solution $\tilde{Y}$ to $B Y=F$ obtained by substitution satisfies

$$
B(\tilde{Y}+\Delta Y)=F+\Delta F, \quad\|\Delta Y\|_{2} \leq \sqrt{n} d_{2} \varepsilon_{M}\|\tilde{Y}\|_{2}, \quad\|\Delta F\|_{2} \leq \sqrt{n} d_{2} \varepsilon_{M}\|F\|_{2},
$$

where $d_{2} \approx 3 n$.

Proof Let $F=\left[f_{1}, f_{2}, \ldots, f_{m}\right]$ and $\tilde{Y}=\left[\tilde{y}_{1}, \tilde{y}_{2}, \ldots, \tilde{y}_{m}\right]$. It is a simple matter (see, e.g. [3]) to prove that the computed solution $\tilde{y}_{j}$ to each system $B y_{j}=f_{j}$ satisfies

$$
B\left(\tilde{y}_{j}+\Delta y_{j}\right)=f_{j}+\Delta f_{j}, \quad j=1, \ldots, m,
$$

where $\left|\Delta y_{j}\right| \leq d_{2} \varepsilon_{M}\left|\tilde{y}_{j}\right|,\left|\Delta f_{j}\right| \leq d_{2} \varepsilon_{M}\left|f_{j}\right|$ and $d_{2} \approx 3 n$.

Thus $B(\tilde{Y}+\Delta Y)=F+\Delta F$, where $\Delta Y$ and $\Delta F$ satisfy the required bounds and the proof is complete.

Lemma 3.3 Let $M \in \mathbb{R}^{n \times n}$ be a symmetric and positive definite matrix and let $F \in$ $\mathbb{R}^{n \times m}$. Then the computed solution $\tilde{X}$ to $M X=F$ obtained via the Cholesky method satisfies

$$
M \tilde{X}=F+\Delta F, \quad\|\Delta F\|_{2} \leq \sqrt{n} d_{3} \varepsilon_{M}\|M\|_{2}\|\tilde{X}\|_{2},
$$

provided that $d_{4} \varepsilon_{M} \kappa_{2}(M)<1$, where $d_{3}$ and $d_{4}$ are small constants depending upon $m$ and $n$. 
Remark 3.1 Here we can apply the result of Higham taking $d_{3} \approx 8 n(n+1)$ and Wilkinson's result for $d_{4} \approx 20 n^{3 / 2}$, see [12, pp. 386-387].

Proof of Lemma 3.3 Let $F=\left[f_{1}, f_{2}, \ldots, f_{m}\right]$ and $\tilde{X}=\left[\tilde{x}_{1}, \tilde{x}_{2}, \ldots, \tilde{x}_{m}\right]$. Then the computed solution $\tilde{x}_{j}$ to each system $M x_{j}=f_{j}$ satisfies

$$
\left(M+\Delta M_{j}\right) \tilde{x}_{j}=f_{j}, \quad \text { where }\left\|\Delta M_{j}\right\|_{2} \leq d_{3} \varepsilon_{M}\|M\|_{2}, j=1, \ldots, m .
$$

From this it follows that $M \tilde{X}=F+\Delta F$, with $\Delta F=-\left[\Delta M_{1} \tilde{x}_{1}, \Delta M_{2} \tilde{x}_{2}, \ldots\right.$, $\left.\Delta M_{m} \tilde{x}_{m}\right]$. Thus

$$
\|\Delta F\|_{2} \leq d_{3} \varepsilon_{M}\|M\|_{2}\left\|\left[\left\|\tilde{x}_{1}\right\|_{2}, \ldots,\left\|\tilde{x}_{m}\right\|_{2}\right]\right\|_{2}=d_{3} \varepsilon_{M}\|M\|_{2}\|\tilde{X}\|_{F} .
$$

Now we use the fact that $\|\tilde{X}\|_{F} \leq \sqrt{n}\|\tilde{X}\|_{2}$ to complete the proof.

Next two lemmas yield backward stability results for Householder $Q R$ factorization and bidiagonal reduction of a matrix of full column rank (see [2, pp. 81-83], [7, p. 124], [3], [12, Chap. 19] and [22]).

Lemma 3.4 Let $\tilde{Q} \tilde{R}$ be the computed thin Householder $Q R$ decomposition of $A \in$ $\mathbb{R}^{m \times n}, m \geq n=\operatorname{rank}(A)$.

Then there exists a left orthogonal matrix $Q \in \mathbb{R}^{m \times n}$ such that

$$
A+\Delta A=Q \tilde{R}, \quad \text { with }\|\Delta A\|_{2} \leq d_{5} \varepsilon_{M}\|A\|_{2},
$$

where $d_{5}$ is a small constant depending upon $m$ and $n$.

Moreover, the computed matrix $\tilde{Q}$ is close to $Q$, i.e.

$$
\tilde{Q}=Q+\Delta Q, \quad \text { where }\|\Delta Q\|_{2} \leq d_{5} \varepsilon_{M} .
$$

Lemma 3.5 Let $\tilde{U} \tilde{B} \tilde{V}^{T}$ be the computed bidiagonal decomposition of $A$ by the Golub-Kahan algorithm for $A \in \mathbb{R}^{m \times n}, m \geq n=\operatorname{rank}(A)$.

Then there exists a left orthogonal matrix $U \in \mathbb{R}^{m \times n}$ and an orthogonal matrix $V \in \mathbb{R}^{n \times n}$ such that

$$
A+\Delta A=U \tilde{B} V^{T}, \quad \text { with }\|\Delta A\|_{2} \leq d_{6} \varepsilon_{M}\|A\|_{2},
$$

where $d_{6}$ is a small constant depending on $m$ and $n$.

Moreover, the computed matrices $\tilde{U}, \tilde{V}$ are close to $U, V$, respectively, i.e.

$$
\tilde{U}=U+\Delta U, \quad\|\Delta U\|_{2} \leq d_{6} \varepsilon_{M}
$$

and

$$
\tilde{V}=V+\Delta V, \quad\|\Delta V\|_{2} \leq d_{6} \varepsilon_{M} .
$$




\subsection{Error analysis of Algorithm I}

Our analysis is similar to this given by Björck [2, pp. 49-50] and Higham [12, pp. 386-387] for the normal equations $A^{T} A x=A^{T} b$.

Theorem 3.1 Let $A \in \mathbb{R}^{m \times n}, m \geq n=\operatorname{rank}(A)$. Assume that

$$
L_{C h o l} \varepsilon_{M} \kappa_{2}^{2}(A)<1,
$$

where

$$
L_{\text {Chol }}=\sqrt{n} d_{3}+m n
$$

and $d_{3} \approx 8 n(n+1)$ (see Lemma 3.3 and Remark 3.1).

Then the computed generalized inverse $\tilde{X}$ obtained by Algorithm I (Chol) satisfies

$$
\frac{\left\|\tilde{X}-A^{\dagger}\right\|_{2}}{\left\|A^{\dagger}\right\|_{2}} \leq L_{C h o l} \varepsilon_{M} \kappa_{2}^{2}(A)+\mathcal{O}\left(\varepsilon_{M}^{2}\right) .
$$

Proof Let $M=A^{T} A$. Since $\operatorname{rank}(A)=n, M$ is nonsingular and we have

$$
A^{\dagger}=M^{-1} A^{T}, \quad\|M\|_{2}=\|A\|_{2}^{2}, \quad\left\|M^{-1}\right\|_{2}=\left\|A^{\dagger}\right\|_{2}^{2},
$$

so $\kappa_{2}(M)=\|M\|_{2}\left\|M^{-1}\right\|_{2}=\kappa_{2}^{2}(A)$.

The matrix $\tilde{M}$ computed by Algorithm I (Chol) satisfies

$$
\tilde{M}=M+\delta M, \quad \text { with }\|\delta M\|_{2} \leq d_{7} \varepsilon_{M}\|M\|_{2},
$$

where $\delta M$ is symmetric and $d_{7} \approx m n$. It is easily seen that

$$
\|\tilde{M}\|_{2} \leq\left(1+d_{7} \varepsilon_{M}\right)\|M\|_{2} .
$$

From (3.2)-(3.3) and Lemma 3.3 it follows that the Cholesky factorization applied to $\tilde{M}$ runs to completion and we get

$$
\tilde{M} \tilde{X}=A^{T}+\Delta A, \quad\|\Delta A\|_{2} \leq \sqrt{n} d_{3} \varepsilon_{M}\|\tilde{M}\|_{2}\|\tilde{X}\|_{2} .
$$

From this and (3.5) we obtain $(M+\delta M) \tilde{X}=A^{T}+\Delta A$, which can be rewritten as $M \tilde{X}=A^{T}+(\Delta A-\delta M \tilde{X})$. Now we premultiply this equation by $M^{-1}$ and use the fact that $A^{\dagger}=M^{-1} A^{T}$ to write

$$
\tilde{X}=A^{\dagger}+\Delta X, \quad \text { where } \Delta X=M^{-1}(\Delta A-\delta M \tilde{X}) .
$$

The next step is to estimate $\|\Delta X\|_{2}$ :

$$
\|\Delta X\|_{2} \leq\left\|M^{-1}\right\|_{2}\left(\|\Delta A\|_{2}+\|\delta M\|_{2}\|\tilde{X}\|_{2}\right) .
$$

This together with (3.5)-(3.7) gives

$$
\|\Delta X\|_{2} \leq\left(\sqrt{n} d_{3}\left(1+d_{7} \varepsilon_{M}\right)+d_{7}\right) \varepsilon_{M}\left\|M^{-1}\right\|_{2}\|M\|_{2}\|\tilde{X}\|_{2} .
$$


Since $\kappa_{2}(M)=\|M\|_{2}\left\|M^{-1}\right\|_{2}=\kappa_{2}^{2}(A)$, we obtain from (3.8) that

$$
\left\|\tilde{X}-A^{\dagger}\right\|_{2}=\|\Delta X\|_{2} \leq\left(\sqrt{n} d_{3}\left(1+d_{7} \varepsilon_{M}\right)+d_{7}\right) \varepsilon_{M} \kappa_{2}^{2}(A)\|\tilde{X}\|_{2} .
$$

Thus, ignoring the terms of order $\mathcal{O}\left(\varepsilon_{M}^{2}\right)$, we conclude that

$$
\frac{\left\|\tilde{X}-A^{\dagger}\right\|_{2}}{\left\|A^{\dagger}\right\|_{2}} \leq\left(\sqrt{n} d_{3}+d_{7}\right) \varepsilon_{M} \kappa_{2}^{2}(A)+\mathcal{O}\left(\varepsilon_{M}^{2}\right),
$$

which establishes (3.4).

Remark 3.2 In Sect. 4 we will give examples showing that Algorithm I (Chol) using the Cholesky decomposition is not mixed forward-backward stable for all matrices. However, Theorem 3.1 indicates that for a narrow class of well-conditioned matrices it may give satisfactory results.

\subsection{Error analysis of Algorithms II-IV}

We now apply Lemmas 3.1 and 3.4 to the computation of the Moore-Penrose inverses by Algorithms II-IV.

Theorem 3.2 Let $A \in \mathbb{R}^{m \times n}$, where $m \geq n=\operatorname{rank}(A)$ and let $\tilde{X}$ be the computed generalized inverse of A obtained in floating point arithmetic by Algorithm II $(Q R)$. Let $\tilde{Q} \tilde{R}$ be the computed thin Householder $Q R$ decomposition of $A$.

Then $\tilde{X}$ satisfies

$$
\frac{\left\|\tilde{X}-A^{\dagger}\right\|_{2}}{\left\|A^{\dagger}\right\|_{2}} \leq L_{Q R} \varepsilon_{M} \kappa_{2}(A)+\mathcal{O}\left(\varepsilon_{M}^{2}\right), \quad L_{Q R}=(\sqrt{2}+1) d_{5}+n d_{1},
$$

provided that

$$
L_{Q R} \varepsilon_{M} \kappa_{2}(A)<1,
$$

where the constants $d_{1}, d_{5}$ are from Lemmas 3.1 and 3.4 , respectively.

Moreover, if there exists a small constant $d_{8}=d_{8}(m, n)$ such that

$$
\text { error } Q R_{Q}=\frac{\left\|\mid \tilde{R}^{-1}\right\| \tilde{R}\|\tilde{X}\|_{2}}{\|\tilde{X}\|_{2}} \leq d_{8},
$$

then Algorithm II is mixed forward-backward stable, i.e. there exist perturbations $\Delta A$ and $\Delta X$ such that $\tilde{X}+\Delta X=(A+\Delta A)^{\dagger}$, where

$$
\|\Delta A\|_{2} \leq d_{5} \varepsilon_{M}\|A\|_{2}, \quad\|\Delta X\|_{2} \leq \frac{\left(d_{5}+d_{1} d_{8}\right) \varepsilon_{M}}{1-d_{5} \varepsilon_{M}}\|\tilde{X}\|_{2} .
$$

Proof By Lemma 3.4 there is a left orthogonal matrix $Q \in \mathbb{R}^{m \times n}$ such that

$$
\begin{aligned}
& A+\Delta A=Q \tilde{R}, \quad\|\Delta A\|_{2} \leq d_{5} \varepsilon_{M}\|A\|_{2}, \\
& \tilde{Q}=Q+\Delta Q, \quad\|\Delta Q\|_{2} \leq d_{5} \varepsilon_{M} .
\end{aligned}
$$


The fact that $d_{5} \varepsilon_{M} \kappa_{2}(A)<1$ implies that $\operatorname{rank}(A+\Delta A)=\operatorname{rank}(A)=n$ (see Lemma 2.2). We have

$$
(A+\Delta A)^{\dagger}=\tilde{R}^{-1} Q^{T}, \quad\left\|\tilde{R}^{-1}\right\|_{2}=\left\|(A+\Delta A)^{\dagger}\right\|_{2}, \quad\|\tilde{R}\|_{2}=\|A+\Delta A\|_{2} .
$$

This together with (2.3)-(2.4) gives

$$
\|\tilde{R}\|_{2} \leq\left(1+d_{5} \varepsilon_{M}\right)\|A\|_{2}, \quad\left\|\tilde{R}^{-1}\right\|_{2} \leq \frac{\left\|A^{\dagger}\right\|_{2}}{1-d_{5} \varepsilon_{M} \kappa_{2}(A)} .
$$

From Lemma 3.1 we have

$$
\tilde{R} \tilde{X}=\tilde{Q}^{T}+\delta Q, \quad|\delta Q| \leq d_{1} \varepsilon_{M}\left|\tilde{R}\|\tilde{X} \mid, \quad\| \delta Q\left\|_{2} \leq n d_{1} \varepsilon_{M}\right\| \tilde{R}\left\|_{2}\right\| \tilde{X} \|_{2}\right.
$$

Thus,

$$
\tilde{X}=\tilde{R}^{-1} \tilde{Q}^{T}+\tilde{R}^{-1} \delta Q
$$

and using (3.13)-(3.14) we get

$$
\tilde{X}+\Delta X=(A+\Delta A)^{\dagger}, \quad \text { where } \Delta X=-\tilde{R}^{-1}\left((\Delta Q)^{T}+\delta Q\right) .
$$

From this and (3.13) and (3.16) we obtain

$$
\|\Delta X\|_{2} \leq d_{5} \varepsilon_{M}\left\|\tilde{R}^{-1}\right\|_{2}+n d_{1} \varepsilon_{M}\|\tilde{R}\|_{2}\left\|\tilde{R}^{-1}\right\|_{2}\|\tilde{X}\|_{2} .
$$

This together with (3.15) gives

$$
\frac{\|\Delta X\|_{2}}{\left\|A^{\dagger}\right\|_{2}} \leq \frac{\varepsilon_{M}}{1-d_{5} \varepsilon_{M} \kappa_{2}(A)}\left(d_{5}+n d_{1}\left(1+d_{5} \varepsilon_{M}\right)\|A\|_{2}\|\tilde{X}\|_{2}\right) .
$$

To bound $\|\tilde{X}\|_{2}$ we use (3.17) and obtain the inequality

$$
\|\tilde{X}\|_{2}=\left\|(A+\Delta A)^{\dagger}-\Delta X\right\|_{2} \leq\left\|(A+\Delta A)^{\dagger}\right\|_{2}+\|\Delta X\|_{2} .
$$

Notice that $\tilde{X}-A^{\dagger}=\left((A+\Delta A)^{\dagger}-A^{\dagger}\right)-\Delta X$. Taking norms and using (2.5), (3.13) and (3.17)-(3.19) we conclude that

$$
\left\|\tilde{X}-A^{\dagger}\right\|_{2} \leq \frac{\sqrt{2} d_{5} \varepsilon_{M} \kappa_{2}(A)\left\|A^{\dagger}\right\|_{2}}{1-d_{5} \varepsilon_{M} \kappa_{2}(A)}+\|\Delta X\|_{2} .
$$

This together with (3.19) gives (3.9), and completes the proof of the first part of the theorem.

To prove the second part of the theorem we use componentwise estimate of $\delta Q$. From (3.16)-(3.17) we have

$$
\|\Delta X\|_{2} \leq\left\|\tilde{R}^{-1}\right\|_{2}\|\Delta Q\|_{2}+\left\|\left|\tilde{R}^{-1}\|\delta Q \mid\|_{2},\right.\right.
$$

so

$$
\|\Delta X\|_{2} \leq d_{5} \varepsilon_{M}\left\|\tilde{R}^{-1}\right\|_{2}+d_{1} \varepsilon_{M}\left\|\left|\tilde{R}^{-1}\|\tilde{R}\| \tilde{X}\right|\right\|_{2} .
$$


This and (3.11) yield

$$
\|\Delta X\|_{2} \leq d_{5} \varepsilon_{M}\left\|\tilde{R}^{-1}\right\|_{2}+d_{1} d_{8} \varepsilon_{M}\|\tilde{X}\|_{2} .
$$

Since $\left\|\tilde{R}^{-1}\right\|_{2}=\left\|(A+\Delta A)^{\dagger}\right\|_{2}=\|\tilde{X}+\Delta X\|_{2} \leq\|\tilde{X}\|_{2}+\|\Delta X\|_{2}$, we see that (3.12) holds. This finishes the proof.

Remark 3.3 Notice that error $_{Q R}$ can be estimated as follows

$$
\operatorname{error}_{Q R} \leq \frac{\left\|\left|\tilde{R}^{-1}\right||\tilde{R}|\right\|_{2}\||\tilde{X}|\|_{2}}{\|\tilde{X}\|_{2}} \leq \sqrt{n} \kappa_{|\tilde{R}|},
$$

where

$$
\kappa_{|\tilde{R}|}=\left\|\left|\tilde{R}^{-1}\right||\tilde{R}|\right\|_{2}
$$

is the Bauer-Skeel condition number. It is known that the Bauer-Skeel (componentwise) condition number can be significantly smaller than the normwise condition number $\kappa_{2}(\tilde{R})=\left\|\tilde{R}^{-1}\right\|_{2}\|\tilde{R}\|_{2} \approx \kappa_{2}(A)$.

The use of column pivoting in $Q R$ factorization can greatly improve the componentwise conditioning of a resulting triangular matrix $\tilde{R}$. In this case we have the inequality

$$
\left|\tilde{r}_{i i}\right| \geq\left|\tilde{r}_{i j}\right|, \quad j>i,
$$

so the unit triangular matrix $W=\left|\tilde{R}^{-1}\right||\tilde{R}|$ satisfies (see Higham [12, p. 143])

$$
w_{i j} \leq 2^{j-i}, \quad j>i
$$

The same holds also for typical algorithms for inverting square matrices by Gaussian Elimination with Complete Pivoting, see [17].

Theorem 3.2 is valid also for Algorithm III. It follows from the error analysis of the $Q R$ with column pivoting (see, e.g., [8]). For discussion on importance of pivoting of the $Q R$ decomposition see the recent paper of Drmač and Bujanović [8]. They stated: 'In particular, pivoting contributes to a more accurate and better-conditioned upper triangular factor'.

From the error analysis of the CGS2 algorithm given by Giraud, Langou, Rozložnik, and Van Den Eshof in [9] it follows that Theorem 3.2 holds for Algorithm IV as well. We have no room for full details here, see [20] and [14].

\subsection{Error analysis of Algorithms V-VII}

Theorem 3.3 Let $A \in \mathbb{R}^{m \times n}, m \geq n=\operatorname{rank}(A)$. Assume that

$$
L_{\text {Bidiag }} \varepsilon_{M} \kappa_{2}(A)<1
$$

where

$$
L_{\text {Bidiag }}=2\left(d_{6}+\sqrt{n} d_{2}+n^{2}\right) .
$$


Then Algorithm $\mathrm{V}\left(\right.$ Bidiag $\left._{1}\right)$ is mixed forward-backward stable, i.e. the computed generalized inverse $\tilde{X}$ satisfies $\tilde{X}+\Delta X=(A+\Delta A)^{\dagger}$, where

$$
\|\Delta A\|_{2} \leq d_{6} \varepsilon_{M}\|A\|_{2}, \quad\|\Delta X\|_{2} \leq L_{\text {Bidiag }}\left(\varepsilon_{M}+\mathcal{O}\left(\varepsilon_{M}^{2}\right)\right)\|\tilde{X}\|_{2} .
$$

Proof By Lemma 3.5 we have

$$
\begin{aligned}
& A+\Delta A=U \tilde{B} V^{T}, \quad\|\Delta A\|_{2} \leq d_{6} \varepsilon_{M}\|A\|_{2}, \\
& \tilde{U}=U+\Delta U, \quad\|\Delta U\|_{2} \leq d_{6} \varepsilon_{M}, \quad \tilde{V}=V+\Delta V, \quad\|\Delta V\|_{2} \leq d_{6} \varepsilon_{M} .
\end{aligned}
$$

From this and Lemma 2.2 it follows that $\operatorname{rank}(A+\Delta A)=\operatorname{rank}(\tilde{B})=n$, and hence

$$
(A+\Delta A)^{\dagger}=V \tilde{B}^{-1} U^{T}, \quad\|A+\Delta A\|_{2}=\|\tilde{B}\|_{2}, \quad\left\|(A+\Delta A)^{\dagger}\right\|_{2}=\left\|\tilde{B}^{-1}\right\|_{2} .
$$

Now, Lemma 3.2 gives us

$$
\tilde{B}(\tilde{Y}+\Delta Y)=\tilde{U}^{T}+\delta U
$$

where

$$
\|\Delta Y\|_{2} \leq \sqrt{n} d_{2} \varepsilon_{M}\|\tilde{Y}\|_{2}, \quad\|\delta U\|_{2} \leq \sqrt{n} d_{2} \varepsilon_{M}\|\tilde{U}\|_{2} .
$$

Using (3.25) we may write (3.28) as

$$
\tilde{Y}+\Delta Y=\tilde{B}^{-1} U^{T}+\tilde{B}^{-1}\left((\Delta U)^{T}+\delta U\right) .
$$

Since $\tilde{V}$ is near orthogonal, we get

$$
\tilde{X}=V \tilde{Y}+\delta X, \quad\|\delta X\|_{2} \leq\left(d_{6}+d_{9}\right) \varepsilon_{M}\|\tilde{X}\|_{2},
$$

where $d_{9} \approx n^{2}$. This together with (3.29) gives

$$
\tilde{X}+\Delta X=(A+\Delta A)^{\dagger}, \quad \Delta X=-V \tilde{B}^{-1}\left((\Delta U)^{T}+\delta U\right)-(\delta X-V \Delta Y) .
$$

We see that from this, (3.24)-(3.27), and (3.22) we obtain the required bound

$$
\|\Delta X\|_{2} \leq L_{\text {Bidiag }}\left(\varepsilon_{M}+\mathcal{O}\left(\varepsilon_{M}^{2}\right)\right)\|\tilde{X}\|_{2} .
$$

The proof is now complete.

Remark 3.4 It is evident that Algorithm VI (Bidiag 2$)$ and Algorithm VII (SVD) are mixed forward-backward stable. Thus Theorem 3.3 is valid also for Algorithms VIVII.

\section{Numerical tests}

In this section we present numerical experiments that show the comparison of Algorithms I-VII. All tests were performed in Matlab, version 6.1.0.450 (R12.1) with machine precision $\varepsilon_{M} \approx 2.2 \times 10^{-16}$.

We considered the following test matrices: 
Table 2 Values of stability factor (4.1) for Algorithms I-VII for computing $X=A^{\dagger}$, where $A \in \mathbb{R}^{n \times n}$ is the Pascal matrix

\begin{tabular}{rllllllll}
\hline$n$ & $\kappa_{2}(A)$ & $e_{C h o l}$ & $e_{Q R}$ & $e_{Q R_{\text {pivot }}}$ & $e_{Q R_{C G S 2}}$ & $e_{\text {Bidiag }}$ & $e_{\text {Bidiag }}$ & $e_{S V D}$ \\
\hline 4 & $6.91 e+2$ & $8.77 e+0$ & $2.60 e-2$ & $3.97 e-2$ & $1.05 e-2$ & $5.92 e-2$ & $3.89 e-2$ & $1.14 e-1$ \\
6 & $1.10 e+5$ & $9.89 e+2$ & $1.80 e-2$ & $2.20 e-3$ & $1.81 e-2$ & $1.04 e-2$ & $1.80 e-2$ & $2.12 e-3$ \\
8 & $2.06 e+7$ & $1.64 e+4$ & $4.53 e-3$ & $1.64 e-3$ & $1.65 e-2$ & $6.24 e-3$ & $4.54 e-3$ & $2.05 e-2$ \\
10 & $4.15 e+9$ & failure & $7.35 e-4$ & $4.33 e-4$ & $7.01 e-3$ & $3.87 e-3$ & $7.35 e-4$ & $3.16 e-3$ \\
\hline
\end{tabular}

Table 3 Values of residual error (4.2) for Algorithms I-VII for computing $X=A^{\dagger}$, where $A \in \mathbb{R}^{n \times n}$ is the Pascal matrix

\begin{tabular}{rllllllll}
\hline$n$ & $\kappa_{2}(A)$ & res Chol & res $Q R$ & res $Q R_{\text {pivot }}$ & res $_{Q R_{C G S 2}}$ & res $_{\text {Bidiag } 1}$ & res Bidiag 2 & res $_{S V D}$ \\
\hline 4 & $6.91 e+2$ & $9.59 e-15$ & $1.93 e-16$ & $3.66 e-17$ & $1.21 e-16$ & $3.82 e-17$ & $4.01 e-17$ & $5.53 e-17$ \\
6 & $1.10 e+5$ & $1.86 e-12$ & $2.61 e-16$ & $1.10 e-17$ & $7.55 e-16$ & $1.35 e-16$ & $2.15 e-17$ & $3.59 e-17$ \\
8 & $2.06 e+7$ & $2.48 e-10$ & $3.87 e-15$ & $2.52 e-17$ & $5.71 e-16$ & $7.96 e-17$ & $2.10 e-17$ & $2.50 e-17$ \\
10 & $4.15 e+9$ & failure & $1.89 e-15$ & $1.03 e-17$ & $9.12 e-15$ & $2.59 e-17$ & $1.26 e-17$ & $1.34 e-17$ \\
\hline
\end{tabular}

1. Pascal matrices:

$$
A=\left(\begin{array}{ccccc}
1 & 1 & 1 & \ldots & 1 \\
1 & 2 & 3 & \ldots & n \\
1 & 3 & 6 & \ldots & a_{3, n-1}+a_{2, n} \\
\vdots & \vdots & \vdots & \ddots & \vdots \\
1 & n & a_{n-1,3}+a_{n, 2} & \ldots & a_{n, n-1}+a_{n-1, n}
\end{array}\right) \in \mathbb{R}^{n \times n}
$$

The entries of the inverse of the Pascal matrix of any size are integer numbers, hence we know the exact result and we can accurately test stability of algorithms.

For each algorithm we computed the following stability factor (compare Definition 2.2)

$$
e_{\text {Algorithm }}=\frac{\left\|\tilde{X}_{\text {Algorithm }}-A^{\dagger}\right\|_{2}}{\varepsilon_{M}\left\|A^{\dagger}\right\|_{2} \kappa_{2}(A)} .
$$

Note that in this case simply $A^{\dagger}=A^{-1}$.

The results are contained in Table 2. For $n=10$ the Cholesky algorithm breaks down. Matrix $A^{T} A$ is not positive definite to working precision.

Table 3 contains values of the following residual error

$$
\text { res }_{\text {Algorithm }}=\frac{\left\|\tilde{X}_{\text {Algorithm }} A-I_{n}\right\|_{2}}{\|A\|_{2}\left\|\tilde{X}_{\text {Algorithm }}\right\|_{2}}
$$

for Pascal matrices (compare Lemma 2.4 and Definition 2.1).

2. Random matrices of entries from the distribution $N(0,1)$. They were generated by Matlab's function "randn". Before each usage the random number generator was reset to its initial state. 
Table 4 Values of stability factor (4.3) for Algorithms I-VI for computing $X=A^{\dagger}$, where $A(3 n \times n)$ is a random matrix

\begin{tabular}{rlllllll}
\hline$n$ & $\kappa_{2}(A)$ & $e_{C h o l}$ & $e_{Q R}$ & $e_{Q R_{\text {pivot }}}$ & $e_{Q R_{C G S 2}}$ & $e_{\text {Bidiag } 1}$ & $e_{\text {Bidiag }}$ \\
\hline 4 & $3.33 e+0$ & $5.84 e+0$ & $5.76 e+0$ & $5.88 e+0$ & $5.81 e+0$ & $5.74 e+0$ & $5.72 e+0$ \\
6 & $2.55 e+0$ & $2.99 e+0$ & $1.91 e+0$ & $2.35 e+0$ & $2.29 e+0$ & $3.26 e+0$ & $3.18 e+0$ \\
8 & $2.73 e+0$ & $2.34 e+0$ & $2.19 e+0$ & $2.28 e+0$ & $2.19 e+0$ & $2.36 e+0$ & $2.33 e+0$ \\
10 & $2.84 e+0$ & $1.87 e+0$ & $1.39 e+0$ & $2.30 e+0$ & $1.83 e+0$ & $2.11 e+0$ & $1.45 e+0$ \\
\hline
\end{tabular}

Table 5 Values of stability factor (4.3) for Algorithms I-VI for computing $X=A^{\dagger}$, where $A \in \mathbb{R}^{n \times n}$ is a random ill-conditioned matrix

\begin{tabular}{rlllllll}
\hline$n$ & $\kappa_{2}(A)$ & $e_{C h o l}$ & $e_{Q R}$ & $e_{Q R_{\text {pivot }}}$ & $e_{Q R} R_{C G S 2}$ & $e_{\text {Bidiag } 1}$ & $e_{\text {Bidiag }}$ \\
\hline 6 & $1.09 e+2$ & $4.68 e+0$ & $4.14 e-2$ & $3.73 e-1$ & $4.32 e-1$ & $5.07 e-1$ & $3.44 e-2$ \\
8 & $2.44 e+1$ & $1.94 e+0$ & $5.00 e-1$ & $5.18 e-1$ & $5.05 e-1$ & $6.72 e-1$ & $4.97 e-1$ \\
10 & $3.25 e+4$ & $1.16 e+3$ & $6.53 e-2$ & $2.38 e-2$ & $1.05 e-1$ & $8.67 e-2$ & $6.52 e-2$ \\
12 & $2.51 e+8$ & $6.21 e+6$ & $3.12 e-1$ & $2.01 e-1$ & $5.16 e-2$ & $7.77 e-2$ & $3.12 e-1$ \\
\hline
\end{tabular}

In this case the exact inverse is not known, so we estimated the stability factor using the SVD algorithm (Algorithm VII, Matlab's function "pinv"). Hence now, instead of formula (4.1), we have

$$
e_{\text {Algorithm }}=\frac{\left\|\tilde{X}_{\text {Algorithm }}-\tilde{X}_{\text {pinv }}\right\|_{2}}{\varepsilon_{M}\left\|\tilde{X}_{\text {pinv }}\right\|_{2} \kappa_{2}(A)} .
$$

Table 4 contains values of this stability factor for random matrices of size $3 n \times n$ for $n=4,6,8,10$. We present the results for matrices of relatively small size, the reason being that neither the condition number nor values of stability factor change significantly with the dimension. The residual error (4.2) in all these tests was of order of machine precision.

3. Ill-conditioned random matrices.

Matrices of random entries of moderate size are in general well-conditioned. To get an ill-conditioned matrix we took $A$ to be the 4th power of a random square matrix. As in the previous test, the distribution was $N(0,1)$ and the random number generator was reset before each usage. Table 5 contains the values of the stability factor (4.3). The values of the residual error (4.2) are gathered in Table 6.

4. This test shows that Algorithms II and IV based on the QR factorization may fail, i.e. the residual error (4.2) can be large. Consequently, Algorithms II and IV are not mixed forward-backward stable for all matrices.

(a) We used very sophisticated example, using so called glued matrices introduced by Smoktunowicz, Barlow and Langou in [21].

Matrix $A$ is defined by the following Matlab code, with different values of the parameter $\mathrm{C}$ : 
Table 6 Values of residual error (4.2) for Algorithms I-VII for computing $X=A^{\dagger}$, where $A \in \mathbb{R}^{n \times n}$ is a random ill-conditioned matrix

\begin{tabular}{rllllllll}
\hline$n$ & $\kappa_{2}(A)$ & res Chol & res $Q R$ & res $Q R_{\text {pivot }}$ & res $_{Q R_{C G S 2}}$ & res $_{\text {Bidiag } 1}$ & res Bidiag & resSVD \\
\hline 6 & $1.09 e+2$ & $1.91 e-15$ & $1.36 e-16$ & $5.88 e-17$ & $1.10 e-16$ & $5.62 e-17$ & $1.41 e-16$ & $1.29 e-16$ \\
8 & $2.44 e+1$ & $2.25 e-15$ & $1.47 e-16$ & $1.94 e-16$ & $1.83 e-16$ & $2.49 e-16$ & $2.06 e-16$ & $3.13 e-16$ \\
10 & $3.25 e+4$ & $1.51 e-12$ & $8.94 e-17$ & $8.34 e-17$ & $2.69 e-17$ & $1.36 e-16$ & $1.10 e-16$ & $4.71 e-17$ \\
12 & $2.51 e+8$ & $1.07 e-8$ & $1.55 e-16$ & $9.55 e-17$ & $1.54 e-16$ & $9.26 e-17$ & $9.89 e-17$ & $7.76 e-17$ \\
\hline
\end{tabular}

Table 7 Values of the error (4.4) for Algorithms II-IV for computing $X=A^{\dagger}$, where $A$ is the $24 \times 12$ matrix defined in $4 \mathrm{a}$

\begin{tabular}{lllll}
\hline $\mathrm{C}$ & $\kappa_{2}(A)$ & error $_{Q R}$ & error $_{Q R_{\text {pivot }}}$ & error $_{Q R_{C G S 2}}$ \\
\hline 1 & $1.44 e+10$ & $3.92 e+2$ & $5.21 e+0$ & $3.92 e+2$ \\
$10^{-1}$ & $1.37 e+10$ & $6.97 e+3$ & $5.66 e+0$ & $6.97 e+3$ \\
$10^{-2}$ & $1.25 e+10$ & $4.30 e+4$ & $5.66 e+0$ & $4.40 e+4$ \\
$10^{-3}$ & $1.29 e+10$ & $4.76 e+5$ & $5.79 e+0$ & $4.76 e+5$ \\
$10^{-4}$ & $1.36 e+10$ & $3.58 e+6$ & $5.81 e+0$ & $3.58 e+6$ \\
$10^{-5}$ & $1.20 e+10$ & $4.23 e+7$ & $5.25 e+0$ & $4.23 e+7$ \\
$10^{-6}$ & $5.58 e+10$ & $1.01 e+8$ & $1.20 e+0$ & $1.01 e+8$ \\
$10^{-8}$ & $6.62 e+12$ & $1.26 e+8$ & $1.55 e+0$ & $1.26 e+8$ \\
\hline
\end{tabular}

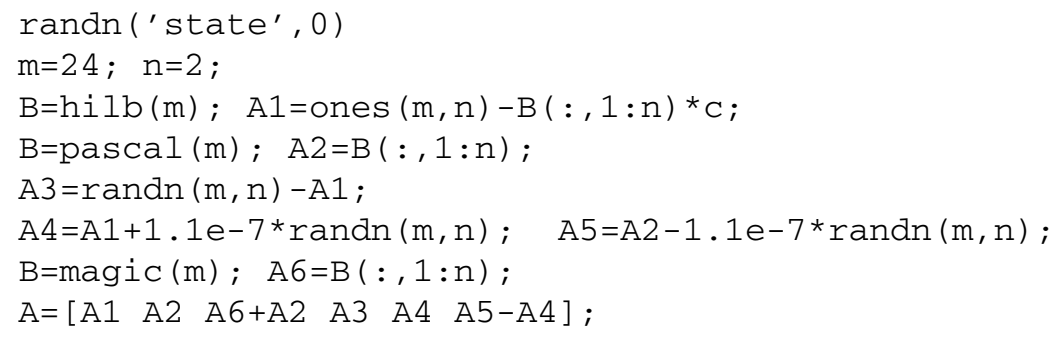

Table 7 contains values of the following error of the $Q R$ factorization

$$
\operatorname{error}_{Q R}=\frac{\left\|| \tilde { R } ^ { - 1 } | \left|\tilde{R}\left\|\tilde{X}_{Q R} \mid\right\|_{2}\right.\right.}{\left\|\tilde{X}_{Q R}\right\|_{2}},
$$

where $\tilde{R}$ is the upper-triangular matrix in the $Q R$ factorization of $A$ and $\tilde{X}_{Q R}$ is the Moore-Penrose inverse computed by Algorithms II-IV. If this error is of order unity, then Algorithms II-IV are mixed forward-backward stable, see Theorem 3.2. In Table 8 we give values of the Bauer-Skeel condition number

$$
\kappa_{|\tilde{R}|}=\left\|\left|\tilde{R}^{-1}\right||\tilde{R}|\right\|_{2} .
$$

Here $\tilde{R}$ is the computed upper-triangular matrix from $Q R$ factorization.

Values of the residual error (4.2) are gathered in Table 9. 
Table 8 Values of the Bauer-Skeel condition number (4.5) for Algorithms II-IV for computing $X=A^{\dagger}$, where $A$ is the $24 \times 12$ matrix defined in $4 \mathrm{a}$

\begin{tabular}{lllll}
\hline $\mathrm{C}$ & $\kappa_{2}(A)$ & $\kappa_{|R|}\left(R_{Q R}\right)$ & $\kappa_{|R|}\left(R_{Q R_{p i v o t}}\right)$ & $\kappa_{|R|}\left(R_{Q R_{C G S 2}}\right)$ \\
\hline 1 & $1.44 e+10$ & $1.80 e+4$ & $6.42 e+0$ & $1.80 e+4$ \\
$10^{-1}$ & $1.37 e+10$ & $1.78 e+5$ & $6.90 e+0$ & $1.78 e+5$ \\
$10^{-2}$ & $1.25 e+10$ & $1.78 e+6$ & $6.83 e+0$ & $1.78 e+6$ \\
$10^{-3}$ & $1.29 e+10$ & $1.78 e+7$ & $5.53 e+0$ & $1.78 e+7$ \\
$10^{-4}$ & $1.36 e+10$ & $1.78 e+8$ & $7.49 e+0$ & $1.78 e+8$ \\
$10^{-5}$ & $1.20 e+10$ & $1.78 e+9$ & $6.74 e+0$ & $1.78 e+9$ \\
$10^{-6}$ & $5.58 e+10$ & $1.78 e+10$ & $7.64 e+0$ & $1.78 e+10$ \\
$10^{-8}$ & $6.62 e+12$ & $1.78 e+12$ & $5.38 e+0$ & $1.78 e+12$ \\
\hline
\end{tabular}

Table 9 Values of residual error (4.2) for Algorithms II-VII for computing $X=A^{\dagger}$, where $A$ is the $24 \times 12$ matrix defined in $4 \mathrm{a}$

\begin{tabular}{llllllll}
\hline $\mathrm{C}$ & $\kappa_{2}(A)$ & res $_{Q R}$ & res $_{Q R_{\text {pivot }}}$ & res $_{Q R_{C G S 2}}$ & res $_{\text {Bidiag }}$ & res $_{\text {Bidiag }}$ & res $_{S V D}$ \\
\hline 1 & $1.44 e+10$ & $5.11 e-15$ & $1.14 e-16$ & $6.39 e-15$ & $1.33 e-16$ & $6.17 e-17$ & $5.39 e-17$ \\
$10^{-1}$ & $1.37 e+10$ & $3.26 e-14$ & $7.75 e-17$ & $5.21 e-14$ & $8.68 e-17$ & $8.15 e-17$ & $4.21 e-17$ \\
$10^{-2}$ & $1.25 e+10$ & $3.55 e-13$ & $2.63 e-17$ & $1.17 e-12$ & $8.14 e-17$ & $1.19 e-16$ & $5.21 e-17$ \\
$10^{-3}$ & $1.29 e+10$ & $4.63 e-12$ & $4.27 e-17$ & $9.25 e-12$ & $1.14 e-16$ & $7.29 e-17$ & $3.88 e-17$ \\
$10^{-4}$ & $1.36 e+10$ & $3.81 e-11$ & $8.02 e-17$ & $3.13 e-11$ & $1.19 e-16$ & $5.77 e-17$ & $5.78 e-17$ \\
$10^{-5}$ & $1.20 e+10$ & $2.58 e-10$ & $1.05 e-16$ & $8.87 e-10$ & $1.50 e-16$ & $9.44 e-17$ & $9.02 e-17$ \\
$10^{-6}$ & $5.58 e+10$ & $4.45 e-10$ & $6.04 e-17$ & $8.73 e-10$ & $6.38 e-17$ & $1.85 e-16$ & $1.44 e-16$ \\
$10^{-8}$ & $6.62 e+12$ & $1.21 e-9$ & $6.09 e-17$ & $3.63 e-10$ & $1.63 e-16$ & $1.40 e-16$ & $1.03 e-16$ \\
\hline
\end{tabular}

Table 10 Values of the error (4.4) for Algorithms II-IV for computing $X=A^{\dagger}$, where $A$ is the $10 \times 10$ matrix defined in $4 \mathrm{~b}$

Table 11 Values of the Bauer-Skeel condition number (4.5) for Algorithms II-IV for computing $X=A^{\dagger}$, where $A$ is the $10 \times 10$ matrix defined in $4 \mathrm{~b}$

\begin{tabular}{llll}
\hline$\kappa_{2}(A)$ & error $_{Q R}$ & error $_{Q R_{\text {pivot }}}$ & error $_{Q R_{C G S 2}}$ \\
\hline $1.22 e+10$ & $3.03 e+5$ & $8.16 e+0$ & $3.03 e+5$ \\
\hline
\end{tabular}

\begin{tabular}{llll}
\hline$\kappa_{2}(A)$ & $\kappa_{|R|}\left(R_{Q R}\right)$ & $\kappa_{|R|}\left(R_{Q R_{\text {pivot }}}\right)$ & $\kappa_{|R|}\left(R_{Q R_{C G S 2}}\right)$ \\
\hline $1.22 e+10$ & $4.77 e+5$ & $9.55 e+0$ & $4.77 e+5$ \\
\hline
\end{tabular}

(b) Now take $A$ generated by the code

$$
\begin{aligned}
& \mathrm{n}=10 ; \\
& \mathrm{A}=\operatorname{inv}(1 e-4 * \operatorname{pascal}(\mathrm{n})+1 e 4 * \operatorname{magic}(\mathrm{n})) ;
\end{aligned}
$$

Tables 10, 11, and 12 contain respective values of the factor (4.4), the Bauer-

Skeel condition number (4.5), and the residual error (4.2).

5. Matrix $A=U S V^{T}$, where $U \in \mathbb{R}^{m \times n}$ and $V \in \mathbb{R}^{n \times n}$ are random (column) orthogonal matrices and $S=\operatorname{diag}\left(1, d, \ldots, d^{n-1}\right)$ with $d=\sqrt{2}$. In this case $A^{\dagger}$ can 
Table 12 Values of residual error (4.2) for Algorithms II-VII for computing $X=A^{\dagger}$, where $A$ is the $10 \times 10$ matrix defined in $4 b$

\begin{tabular}{lllllll}
\hline$\kappa_{2}(A)$ & res $Q R$ & res $_{Q R}$ pivot & res $_{Q R} R_{C S 2}$ & res $_{\text {Bidiag }}$ & res $_{\text {Bidiag }}$ & res $_{S V D}$ \\
\hline $1.22 e+10$ & $2.32 e-12$ & $7.97 e-17$ & $1.95 e-12$ & $1.56 e-16$ & $1.88 e-16$ & $8.53 e-17$ \\
\hline
\end{tabular}

Table 13 Values of stability factor (4.1) for Algorithms I-VII for computing $X=A^{\dagger}$, where $A$ is the matrix defined in 5

\begin{tabular}{rllllllll}
\hline$n$ & $\kappa_{2}(A)$ & $e_{C h o l}$ & $e_{Q R}$ & $e_{Q R_{\text {pivot }}}$ & $e_{Q R_{C G S 2}}$ & $e_{\text {Bidiag }}$ & $e_{\text {Bidiag } 2}$ & $e_{S V D}$ \\
\hline 10 & $2.26 e+1$ & $1.98 e+0$ & $8.35 e-1$ & $4.62 e-1$ & $3.26 e-1$ & $4.75 e-1$ & $8.36 e-1$ & $8.69 e-1$ \\
25 & $4.09 e+3$ & $2.18 e+2$ & $5.40 e-1$ & $2.99 e-1$ & $1.67 e-1$ & $3.73 e-1$ & $5.40 e-1$ & $5.40 e-1$ \\
40 & $7.41 e+5$ & $5.92 e+4$ & $6.04 e-1$ & $3.83 e-1$ & $2.24 e-1$ & $2.52 e-1$ & $6.04 e-1$ & $6.04 e-1$ \\
55 & $1.34 e+8$ & $5.51 e+6$ & $6.99 e-1$ & $6.39 e-1$ & $1.87 e-1$ & $2.53 e-1$ & $6.99 e-1$ & $6.99 e-1$ \\
70 & $2.42 e+10$ & failure & $2.89 e-1$ & $1.48 e-1$ & $1.91 e-1$ & $4.09 e-1$ & $2.89 e-1$ & $2.89 e-1$ \\
85 & $4.39 e+12$ & failure & $3.18 e-1$ & $2.17 e-1$ & $2.03 e-1$ & $2.65 e-1$ & $3.18 e-1$ & $3.18 e-1$ \\
100 & $7.96 e+14$ & failure & $2.35 e-1$ & $2.62 e-1$ & $2.03 e-1$ & $3.82 e-1$ & $2.35 e-1$ & $5.66 e+0$ \\
\hline
\end{tabular}

Table 14 Values of residual error (4.2) for Algorithms I-VII for computing $X=A^{\dagger}$, where $A$ is the matrix defined in 5

\begin{tabular}{rllllllll}
\hline$n$ & $\kappa_{2}(A)$ & res Chol & res $_{Q R}$ & res $_{Q R_{\text {pivot }}}$ & res $_{Q R_{C G S 2}}$ & res $_{\text {Bidiag }}$ & res $_{\text {Bidiag }}$ & resSVD \\
\hline 10 & $2.26 e+1$ & $8.44 e-16$ & $1.78 e-16$ & $7.51 e-17$ & $6.37 e-17$ & $1.37 e-16$ & $2.94 e-16$ & $2.05 e-16$ \\
25 & $4.09 e+3$ & $2.79 e-13$ & $1.07 e-16$ & $7.13 e-17$ & $7.69 e-17$ & $1.29 e-16$ & $6.75 e-17$ & $7.17 e-17$ \\
40 & $7.41 e+5$ & $5.21 e-11$ & $9.46 e-17$ & $6.76 e-17$ & $1.02 e-16$ & $7.32 e-17$ & $4.97 e-17$ & $4.42 e-17$ \\
55 & $1.34 e+8$ & $8.98 e-9$ & $1.13 e-16$ & $8.19 e-17$ & $1.20 e-16$ & $1.48 e-16$ & $8.05 e-17$ & $9.12 e-17$ \\
70 & $2.42 e+10$ & failure & $1.71 e-16$ & $7.07 e-17$ & $1.91 e-16$ & $1.09 e-16$ & $5.13 e-17$ & $4.90 e-17$ \\
85 & $4.39 e+12$ & failure & $2.13 e-16$ & $5.91 e-17$ & $3.43 e-16$ & $1.12 e-16$ & $9.79 e-17$ & $8.55 e-17$ \\
100 & $7.96 e+14$ & failure & $1.97 e-16$ & $1.20 e-16$ & $2.36 e-16$ & $1.17 e-16$ & $7.94 e-17$ & $1.14 e-13$ \\
\hline
\end{tabular}

be computed with high accuracy as $A^{\dagger}=V S^{-1} U^{T}$. Notice that here $\left\|A^{\dagger}\right\|_{2}=1$ and $\kappa_{2}(A)=d^{n-1}$.

Table 13 contains values of the stability factor (4.1). For these tests we took $m=5 n$. Values of the residual error (4.2) are gathered in Table 14.

6. The Kahan matrix (see $[18$, p. 31$]$ or $[10$, p. 260$]$ ):

$$
T_{n}(c)=\operatorname{diag}\left(1, s, s^{2}, \ldots, s^{n-1}\right)\left(\begin{array}{ccccc}
1 & -c & -c & \ldots & -c \\
0 & 1 & -c & \ldots & -c \\
\vdots & \vdots & \ddots & \ddots & \vdots \\
0 & 0 & \ldots & 1 & -c \\
0 & 0 & \ldots & 0 & 1
\end{array}\right)
$$


Table 15 Values of the Bauer-Skeel condition number (4.5) for Algorithms II-IV for computing $X=A^{\dagger}$, where $A(100 \times 100)$ is the Kahan matrix (4.6)

\begin{tabular}{lllll}
\hline$c$ & $\kappa_{2}(A)$ & $\kappa_{|R|}\left(R_{Q R}\right)$ & $\kappa_{|R|}\left(R_{Q R_{p i v o t}}\right)$ & $\kappa_{|R|}\left(R_{Q R_{C G S 2}}\right)$ \\
\hline 0.10 & $5.42 e+4$ & $1.31 e+4$ & $1.05 e+1$ & $1.31 e+4$ \\
0.15 & $1.13 e+7$ & $1.09 e+6$ & $1.49 e+1$ & $1.09 e+6$ \\
0.20 & $2.18 e+9$ & $7.53 e+7$ & $2.41 e+1$ & $7.53 e+7$ \\
0.25 & $4.37 e+11$ & $4.36 e+9$ & $1.82 e+1$ & $4.36 e+9$ \\
0.30 & $9.77 e+13$ & $2.16 e+11$ & $3.33 e+1$ & $2.16 e+11$ \\
0.35 & $2.57 e+16$ & $9.19 e+12$ & $2.61 e+1$ & $9.19 e+12$ \\
0.40 & $8.39 e+18$ & $3.42 e+14$ & $2.58 e+1$ & $3.42 e+14$ \\
\hline
\end{tabular}

Table 16 Values of residual error (4.2) for Algorithms II-VII for computing $X=A^{\dagger}$, where $A(100 \times 100)$ is the Kahan matrix (4.6)

\begin{tabular}{llllllll}
\hline$c$ & $\kappa_{2}(A)$ & ${ }^{r e s} Q R$ & res $_{Q R}{ }_{\text {pivot }}$ & res $_{Q R}{ }_{C G S 2}$ & res $_{\text {Bidiag }}$ & res $_{\text {Bidiag }}$ & res $_{S V D}$ \\
\hline 0.10 & $5.42 e+4$ & $1.39 e-17$ & $8.09 e-17$ & $1.39 e-17$ & $2.83 e-16$ & $2.83 e-16$ & $1.50 e-16$ \\
0.15 & $1.13 e+7$ & $5.24 e-18$ & $1.84 e-17$ & $5.24 e-18$ & $9.81 e-17$ & $9.81 e-17$ & $8.61 e-17$ \\
0.20 & $2.18 e+9$ & $2.46 e-18$ & $6.87 e-18$ & $2.46 e-18$ & $1.34 e-16$ & $1.34 e-16$ & $6.89 e-17$ \\
0.25 & $4.37 e+11$ & $1.24 e-18$ & $3.86 e-18$ & $1.24 e-18$ & $1.07 e-16$ & $1.07 e-16$ & $6.49 e-17$ \\
0.30 & $9.77 e+13$ & $2.18 e-19$ & $3.12 e-19$ & $2.18 e-19$ & $1.10 e-17$ & $1.10 e-17$ & $1.24 e-3$ \\
0.35 & $2.57 e+16$ & $4.92 e-20$ & $7.41 e-20$ & $4.92 e-20$ & $1.66 e-18$ & $1.66 e-18$ & $2.07 e-4$ \\
0.40 & $8.39 e+18$ & $3.07 e-21$ & $4.80 e-21$ & $3.07 e-21$ & $1.11 e-19$ & $1.11 e-19$ & $2.44 e-5$ \\
\hline
\end{tabular}

where $s^{2}+c^{2}=1$ with $s, c>0$. Depending on the choice of $s$ and $c$ this matrix can be very ill-conditioned. It is interesting that in this case the Bauer-Skeel condition number $\kappa_{\left|T_{n}(c)\right|}=\left\|\left|T_{n}(c)^{-1}\right|\left|T_{n}(c)\right|\right\|_{2}$ is also large.

Tables 15 and 16 contain respective values of the Bauer-Skeel condition number (4.5) and the residual error (4.2).

Notice that for $c=0.3$ and larger the residuum for the SVD algorithm is very large! Similar results are obtained for $c \geq 0.4$. The conditioning worsens with the growth of $c$, however the pattern is the same: the SVD algorithm gives significantly worse results than the other algorithms.

\section{Conclusions}

- Algorithm I obtained via the Cholesky decomposition is the cheapest method, however, it is not suitable for every matrix. The accuracy depends on the square of the condition number instead of the condition number $\left(\kappa_{2}^{2}(A)\right.$ instead of $\left.\kappa_{2}(A)\right)$. Thus the error is always bounded by $\varepsilon_{M} \kappa_{2}^{2}(A)$. Since the normal equations are the fastest way to compute the pseudoinverse, they may be a method of choice, but only if the matrix $A$ is well-conditioned.

- Algorithms II-VII are forward stable. 
- Algorithms II and IV are not mixed forward-backward stable but only conditionally mixed forward-backward stable.

- The superiority of use of the $Q R$ factorization with column pivoting for computing the Moore-Penrose inverse over $Q R$ decomposition (without pivoting) was experimentally confirmed (see Sect. 4).

- Algorithm V is a generalization of the algorithm proposed by Byers and $\mathrm{Xu}$ in [3]. It has the same numerical properties (mixed forward-backward stability) as Algorithm VII (based on the SVD decomposition). It is less expensive in comparison with Algorithm VII and can be used for computing $A^{\dagger}$ if the matrix $A$ is not rank deficient. In this case one can avoid an implicit-shift $Q R$ step applied to the tridiagonal matrix in the SVD algorithm, using only the factors from bidiagonalization, with significantly less number of floating point operations in comparison with the SVD algorithm.

Acknowledgements We are grateful to the referees for their suggestions, which helped to improve this paper. We also thank Jesse L.Barlow for his implementation of bidiagonalization.

Open Access This article is distributed under the terms of the Creative Commons Attribution Noncommercial License which permits any noncommercial use, distribution, and reproduction in any medium, provided the original author(s) and source are credited.

\section{References}

1. Abdelmalek, N.I.: Roundoff error analysis for Gram-Schmidt method and solution of linear least squares problems. BIT 11(4), 354-367 (1971)

2. Björck, Å.: Numerical Methods for Least Squares Problems. SIAM, Philadelphia (1996)

3. Byers, R., Xu, H.: A new scaling for Newton's iteration for the polar decomposition and its backward stability. SIAM J. Matrix Anal. Appl. 30, 822-843 (2008)

4. Chan, T.F.: An improved algorithm for computing the singular value decomposition. ACM Trans. Math. Softw. 8(1), 72-83 (1982)

5. Chan, T.F.: Rank revealing $Q R$-factorizations. Linear Algebra Appl. 88/89, 67-82 (1987)

6. Courrieu, P.: Fast computation of Moore-Penrose inverse matrices. Neural Inform. Process. Lett. Rev. 8(2), 25-29 (2005)

7. Demmel, J.W.: Applied Numerical Linear Algebra. SIAM, Philadelphia (1997)

8. Drmač, Z., Bujanović, Z.: On the failure of rank-revealing $Q R$ factorization software-a case study. ACM Trans. Math. Softw. 35(2), 1-28 (2008)

9. Giraud, L., Langou, J., Rozložnik, M., Van Den Eshof, J.: Rounding error analysis of the classical Gram-Schmidt orthogonalization process. Numer. Math. 101(1), 87-100 (2005)

10. Golub, G.H., Van Loan, Ch.F.: Matrix Computations, third edn. Johns Hopkins University Press, Baltimore (1996)

11. Higham, N.J.: $Q R$ factorization with complete pivoting and accurate computation of the SVD. Linear Algebra Appl. 309, 153-174 (2000)

12. Higham, N.J.: Accuracy and Stability of Numerical Algorithms, second edn. SIAM, Philadelphia (2002)

13. Higham, N.J.: Functions of Matrices. Theory and Computation. SIAM, Philadelphia (2008)

14. Hoffmann, W.: Iterative algorithms for Gram-Schmidt orthogonalization. Computing 41, 353-367 (1989)

15. Kaczorek, T.: Wektory i Macierze w Automatyce i Elektrotechnice. WNT, Warszawa (1998) (in Polish)

16. Katsikis, V.N., Pappas, D.: Fast computing of the Moore-Penrose inverse matrix. Electron. J. Linear Algebra 17, 637-650 (2008) 
17. Kiełbasiński, A., Zieliński, P., Ziętak, K.: Higham's scaled method for polar decomposition and numerical matrix-inversion. Technical report Institute of Mathematics and Computer Science Report I18/2007/P-045, Wroclaw University of Technology, Wroclaw, Poland (2007)

18. Lawson, C.L., Hanson, R.J.: Solving Least Squares Problems. Prentice-Hall, Englewood Cliffs (1974)

19. Petković, M., Stanimirović, P.: Generalized inversion is not harder than matrix multiplication. J. Comput. Appl. Math. 230(1), 270-282 (2009)

20. Ruhe, A.: Numerical aspects of Gram-Schmidt orthogonalization of vectors. Linear Algebra Appl. 52/53, 591-601 (1983)

21. Smoktunowicz, A., Barlow, J.L., Langou, J.: A note on the error analysis of classical Gram-Schmidt. Numer. Math. 105(2), 299-313 (2006)

22. Wilkinson, J.H.: The Algebraic Eigenvalue Problem. Oxford University Press, London (1965) 